\title{
Avaliação do nível de conhecimento em relação à Aids e sífilis por idosos do interior cearense, Brasil
}

\author{
Evaluation of the level of knowledge about Aids and syphilis among \\ the elderly from a city in the interior of the state of Ceará, Brazil
}

\author{
Luzia Mesquita Bastos ${ }^{1}$ \\ Jéssika Mayhara Souza Tolentino ${ }^{1}$ \\ Maria Alanne de Oliveira Frota ${ }^{1}$ \\ Wellington Costa Tomaz ${ }^{1}$ \\ Maria Luisa de Sousa Fialho ${ }^{2}$ \\ Ana Cristina Beviláqua Batista ${ }^{3}$ \\ Ana Karine Macedo Teixeira ${ }^{4}$ \\ Francisco Cesar Barroso Barbosa ${ }^{1}$
}

${ }^{1}$ Universidade Federal do Ceará. R. Coronel Estanislau Frota s/n, Centro. 62010560 Sobral CE Brasil. luziambastos@hotmail.com ${ }^{2}$ Centro de Saúde da Família Estaç̃o, Prefeitura Municipal de Sobral. Sobral CE Brasil.

${ }^{3}$ Centro de Especialidades Odontológicas Regional de Sobral. Sobral CE Brasil.

${ }^{4}$ Faculdade de Farmácia, Odontologia e Enfermagem, Universidade Federal do Ceará. Fortaleza CE Brasil.

\begin{abstract}
The scope of this study was to evaluate the knowledge of the elderly about syphilis and AIDS before and after conducting educational activities. Fifty-five individuals participating in a convenience group from territory of Terrenos Novos in Sobral in the State of Ceará were selected. To collect the data, a semi-structured questionnaire about HIV/AIDS and syphilis was used, including general features and information about concept, transmission, prevention, vulnerability and treatment. The questionnaire was applied before and after the implementation of educational workshops about AIDS and syphilis. Among those surveyed, $96.4 \%$ were aware about AIDS and $67.3 \%$ did not know about syphilis. However, $38.1 \%$ believed that a kiss on the mouth and $78.1 \%$ that mosquito bites were means of HIV transmission. $70.9 \%$ did not know the mode of transmission of syphilis. After the workshops the indexes changed positively. Data analysis revealed gaps in knowledge of this group about the concept, transmission and treatment of these diseases. Educational interventions were effective for knowledge assimilation.
\end{abstract}

Key words Perception, Elderly, AIDS, Syphilis
Resumo O propósito deste trabalho foi avaliar o conhecimento de idosos acerca da sífilis e Aids antes e após a realização de ações educativas. Foram selecionados 55 idosos que participavam de grupo de conveniência no território dos Terrenos Novos, em Sobral - CE. Para a coleta dos dados, utilizouse um questionário semiestruturado sobre HIV/ Aids e sifilis, incluindo características gerais e informações sobre conceito, transmissão, prevenção, vulnerabilidade e tratamento. O questionário foi aplicado antes e após a realização de oficinas educativas sobre Aids e sifilis. Dentre os investigados, 96,4\%, tinham conhecimento sobre Aids e 67,3\% desconheciam a sifilis. Entretanto, 38,1\% acreditavam que beijo na boca e $78,1 \%$ que picada de mosquito eram meios de transmissão do HIV. Desconheciam a forma de transmissão da sífilis 70,9\%. Após as oficinas os indices alteraram positivamente. A análise dos dados revelou lacunas no conhecimento deste grupo quanto ao conceito, transmissão e tratamento dessas doenças. As intervenções educativas mostraram-se eficazes para a assimilação de conhecimento.

Palavras-chave Percepção, Idosos, Aids, Sífilis 


\section{Introdução}

Nos últimos anos, observa-se uma inversão da pirâmide etária caracterizada pelo aumento significativo da população idosa, aliado ao crescimento da expectativa de vida deste grupo ${ }^{1}$. Segundo dados do $\mathrm{IBGE}^{2}$, em 2012, o número de idosos no Brasil correspondia a cerca de 12,6\% da população total. Além disso, a expectativa de vida do brasileiro também aumentou, atingindo em 2012 a média de 74,6 anos. Estimativas apontam que em 2025, o Brasil será o sexto país no mundo em população idosa ${ }^{1,3,4}$. De acordo com a Organização Mundial da Saúde ${ }^{5}$, nos países em desenvolvimento, considera-se terceira idade os indivíduos a partir dos 60 anos.

O envelhecimento é um processo dinâmico, sequencial, individual e não patológico. Nele se observa modificações morfológicas, funcionais, bioquímicas e psicológicas. O crescimento senil associado à melhoria da qualidade de vida implica numa nova tendência de aumento da proliferação de doenças infectocontagiosas nessa faixa etária, tais como as Infecções Sexualmente Transmissíveis (IST), em especial a Aids e a sífilis ${ }^{6,7}$.

A Aids é considerada uma doença emergente, grave e de comportamento pandêmico. Desde o início da epidemia no Brasil, em 1980, até junho de 2015, foram registrados no país 798.366 casos de Aids sendo 65,0\% casos em homens e 35,0\% em mulheres ${ }^{8}$. Embora a maioria dos casos de infecção pelo HIV seja detectada na faixa etária de 25 a 39 anos, têm sido verificadas mudanças no seu perfil epidemiológico e o aumento do número de infectados na faixa acima de 50 anos. No Ceará, desde o primeiro caso conhecido em 1983, foram notificados 13.208 casos até novembro de 2013, no Sistema de Informação de Agravos de Notificação (SINAN) ${ }^{9}$. A taxa de incidência de pessoas com mais de 50 anos infectados aumentou de 4,1/100.000 habitantes, em 2001, para 9,3/100.000 habitantes, $2012^{9}$.

A sífilis é uma doença de evolução crônica, que vem surgindo mais frequentemente nos últimos anos. Estima-se que, no Brasil, sejam infectados todos os anos em torno de 937 mil pessoas ${ }^{10}$. Conforme dados do Ministério da Saúde a região Nordeste registrou 28,9\%, que corresponde a 11.905 casos $^{11}$.

O aumento da expectativa e da qualidade de vida, bem como avanços na medicina, na indústria farmacêutica e a disponibilidade de medicamentos que permitem o prolongamento da vida sexual ocasionaram mudanças no comportamento sexual do idoso, tornando essa faixa etária mais vulnerável às IST ${ }^{12-14}$.
Dentre os fatores associados, destacam-se: a resistência ao uso de preservativos, o pouco conhecimento dos idosos acerca de práticas de sexo seguro e em relação às IST $^{12,15}$. Além disso, as campanhas preventivas para Aids normalmente são voltadas para o público mais jovem, esta negligência em relação à terceira idade, pode fazer desta faixa etária, um grupo potencialmente de risco $^{16}$.

Nesse contexto, pesquisas sobre IST se fazem necessárias, principalmente com idosos, já que estudos nessa faixa etária são limitados, devido à questão da sexualidade na terceira idade ainda ser tratada como tabu por muitos, até mesmo por profissionais de saúde.

Desta forma, o propósito deste estudo foi avaliar o conhecimento de idosos acerca da sífilis e HIV antes e após a realização de ações educativas.

\section{Metodologia}

Trata-se de um estudo quantitativo de intervenção, que permitiu avaliar o nível de conhecimento dos idosos acerca de Aids e sífilis. Foi realizado por preceptores enfermeiros e dentistas, além de monitores do Programa de Educação pelo Trabalho para a Saúde (PET- Saúde), acadêmicos dos cursos de Medicina, Odontologia e Psicologia da Universidade Federal do Ceará/Campus Sobral, no bairro dos Terrenos Novos, no município de Sobral, Ceará.

Foram selecionados idosos, com idade igual ou superior a 60 anos, de ambos os sexos, que participavam de grupos de convivência organizados pela equipe de saúde da família do território dos Terrenos Novos no município de Sobral, Ceará. A amostra foi composta por 55 idosos, correspondente a $10 \%$ da população senil cadastrada e acompanhada no Centro de Saúde da Família dos Terrenos Novos. Foram adotados como critérios de exclusão os idosos restritos ao lar.

A coleta de dados foi realizada no período de Janeiro a Março de 2014 por meio da aplicação de um questionário objetivo, composto por 14 questões. $\mathrm{O}$ instrumento foi adaptado a partir do questionário estruturado e validado por Lazzarotto et al. sobre HIV para terceira idade (QHIV3I). No questionário foram abordadas informações socioeconômicas, gênero, idade, estado civil e aspectos tais como transmissibilidade, grupos de risco, prevenção e tratamento, relacionados à HIV/Aids e sífilis.

Os dados foram coletados antes e logo após a realização de oficinas educativas. As oficinas 
eram realizadas semanalmente por 4 monitores do PET-Saúde, acadêmicos dos cursos de Medicina, Odontologia e Psicologia, que atuavam como facilitadores. Foram utilizados recursos lúdicos e didáticos como slides e placas ilustrativas confeccionados pelos monitores, além de cadernos ilustrativos sobre Aids e sífilis disponibilizados pelo ministério da saúde. Tais recursos tinham como objetivo estimular a participação dos idosos, de tal maneira que não fossem expectadores, mas também atores no processo de aprendizagem. Nas oficinas foram discutidos assuntos relacionados ao conhecimento, formas de transmissão, grupos de risco, prevenção e tratamento de Aids e sífilis, e também foram esclarecidas dúvidas dos participantes, que surgiram ao longo da atividade.

Para a avaliação do nível de conhecimento foi estabelecido um indicador de conhecimento referente ao número de acertos que o participante tinha ao longo do questionário, tendo variação de 0-20 pontos, sendo, quanto maior o número de pontos obtidos maior o nível de conhecimento.

Os dados dos questionários foram convertidos para o programa Excel 2007, tabulados e armazenados. Em seguida, as avaliações dos resultados foram analisadas estatisticamente pelos testes $\mathrm{t}$ e $\mathrm{t}$ pareado, com auxílio do programa SPSS versão 20, tendo significância menor que $5 \%(\mathrm{p}<0,05)$.

O estudo foi submetido à avaliação do Comitê de Ética em Pesquisa da Universidade Estadual do Vale do Acaraú (UVA), tendo sido aprovado. Durante o desenvolvimento da pesquisa foram seguidos os princípios éticos, de acordo com a Resolução no 466/2012 do Conselho Nacional de Saúde, sobre pesquisa científica desenvolvidas com seres humanos. Todos os envolvidos aceitaram participar voluntariamente após a leitura e assinatura do Termo de Consentimento Livre e Esclarecido, onde foram esclarecidos sobre a finalidade e passos metodológicos do estudo.

\section{Resultados}

Foram entrevistados 55 indivíduos, de ambos os sexos, com idade igual ou superior a 60 anos. As mulheres correspondiam à maioria da população entrevistada (74,5\%). A idade variava de 60 a 90 anos, com 69\% da amostra na faixa etária entre 60 e 70 anos. Em relação à renda mensal, observou-se que a maioria dos idosos recebia entre 1 e 2 salários mínimos $(78,1 \%)$, e quanto à atividade ocupacional, 87,3\% eram aposentados e recebiam benefícios mensais. No que concerne à relação conjugal, os casados ou que possuíam companheiros fixos correspondia à maioria (58,2\%) (Tabela 1).

Quando questionados sobre a atividade sexual, apenas 30,9\% dos entrevistados referiram ter vida sexual ativa à época da entrevista. No entanto, quando indagados se usavam ou se já usaram preservativos durante a relação sexual, $90,9 \%$ afirmaram que não. Foi possível perceber que não houve diferença estatisticamente significante entre a média de conhecimento entre os sexos, bem como entre casados e não casados. Já em relação ao uso de preservativo, os dados demonstraram que aqueles que o usam têm média de conhecimento maior $(\mathrm{p}=0,03)$. O mesmo foi observado em relação à vida sexual ativa, sendo que o idoso sexualmente ativo também tem média de conhecimento maior.

Em relação ao conhecimento da Aids, 96,4\% referiram já ter conhecimento sobre a doença, seja por meio de informativos, meios de comunicação ou campanhas de saúde pública. Ao serem questionados quanto às vias de transmissão, $12,7 \%$ afirmaram não saber como esta se faz, entretanto o índice, após a intervenção educativa, reduziu para zero. Dos idosos entrevistados, $78,1 \%$ acreditava que o vírus HIV poderia ser

Tabela 1. Descrição da amostra do estudo $(n=55)$ em Sobral, CE.

\begin{tabular}{|c|c|c|}
\hline Variáveis & $\mathbf{n}$ & $\%$ \\
\hline \multicolumn{3}{|l|}{ Gênero } \\
\hline Feminino & 41 & $74,5 \%$ \\
\hline Masculino & 14 & $25,5 \%$ \\
\hline \multicolumn{3}{|l|}{ Faixa etária } \\
\hline $60-70$ & 38 & $69,0 \%$ \\
\hline $71-80$ & 14 & $25,5 \%$ \\
\hline Acima de 80 & 03 & $5,5 \%$ \\
\hline \multicolumn{3}{|l|}{ Estado civil } \\
\hline Casado & 32 & $58,2 \%$ \\
\hline Não casado & 23 & $41,8 \%$ \\
\hline \multicolumn{3}{|l|}{ Atividade Ocupacional } \\
\hline Aposentado & 48 & $87,3 \%$ \\
\hline Desempregado & 04 & $7,2 \%$ \\
\hline \multirow[t]{2}{*}{ Trabalho autônomo } & 03 & \\
\hline & & $5,5 \%$ \\
\hline \multicolumn{3}{|l|}{ Renda Mensal } \\
\hline Sem renda & 06 & $10,9 \%$ \\
\hline$<1$ salário $^{*}$ & 03 & $5,5 \%$ \\
\hline Entre 1 e 2 salários & 43 & $78,1 \%$ \\
\hline$>2$ salários & 03 & $5,5 \%$ \\
\hline
\end{tabular}

*1 salário mínimo em 2014 correspondia a R\$724,00. 
transmitido através da picada de mosquito, após a oficina esse número reduziu para $23,6 \%$. Resultados similares e expressivos, como pode ser observado no Gráfico 1, foram encontrados quando questionados sobre o uso do mesmo sanitário, uso do mesmo copo e beijo na boca. Já quanto indagados sobre o sexo sem preservativo, $78,1 \%$ afirmaram que era uma forma de transmissão do vírus HIV, após a intervenção educativa o número aumentou para $100 \%$. A mesma tendência de resultados ocorreu em relação aos quesitos, sexo oral e aleitamento materno (Gráfico 1).

Em relação à prevenção da Aids, 61,8\% dos idosos afirmaram saber como se proteger. Desse total, $90,24 \%$ indicaram a camisinha como meio de prevenção, $60,97 \%$ o uso de vacinas. Após a intervenção educativa $100 \%$ indicaram apenas o uso de preservativo como meio de prevenção.

Quando questionados sobre sífilis, 67,3\% dos idosos entrevistados afirmaram que desconheciam a doença, enquanto que $70,9 \%$ afirmaram não saber sua forma de transmissão. Dos demais, quando indagados sobre as vias de transmissibilidade, $7,3 \%$ acreditavam que dormir no mesmo quarto era uma via de transmissão, após a oficina o índice diminuiu para 1,8\%. Resultados similares foram obtidos quando indagados sobre o uso do mesmo copo e picada de mosquito. Quanto ao sexo sem preservativo, 30,9\% afirmaram ser forma de transmissão da sífilis, após a atividade educativa o número subiu para $98,1 \%$; já em relação ao sexo oral o número aumentou de $21,8 \%$ para $87,2 \%$ (Tabela 2).

Em relação à forma de prevenção da sífilis, antes da intervenção educativa apenas $83,6 \%$ dos idosos afirmaram não saber como se prevenir. Quanto à cura para a sífilis, 25,4\% acreditavam que a doença tinha tratamento e que o paciente ficava curado e $56,4 \%$ não sabiam responder. Após a intervenção $87,2 \%$ afirmaram que a sífilis tinha tratamento e cura.

Quando indagados se apenas os mais jovens estavam vulneráveis em adquirir tais doenças os resultados foram diferentes. Quanto à Aids, $81,8 \%$ dos idosos acreditavam que todas as faixas etárias estavam vulneráveis, após a intervenção, o número aumentou para $100 \%$. Em relação à sífilis, 56,3\% acreditavam que a doença era uma preocupação de todas as faixas etárias e $38,1 \%$ não sabiam responder. Após as oficinas 96,3\% passaram a afirmar que todos estavam susceptíveis a sífilis.

Na Tabela 3, estão descritas as comparações das médias de conhecimento dos idosos antes e após as oficinas. Foi possível observar um aumento significativo de 11,7 para $17,3(\mathrm{p}<0,001)$.

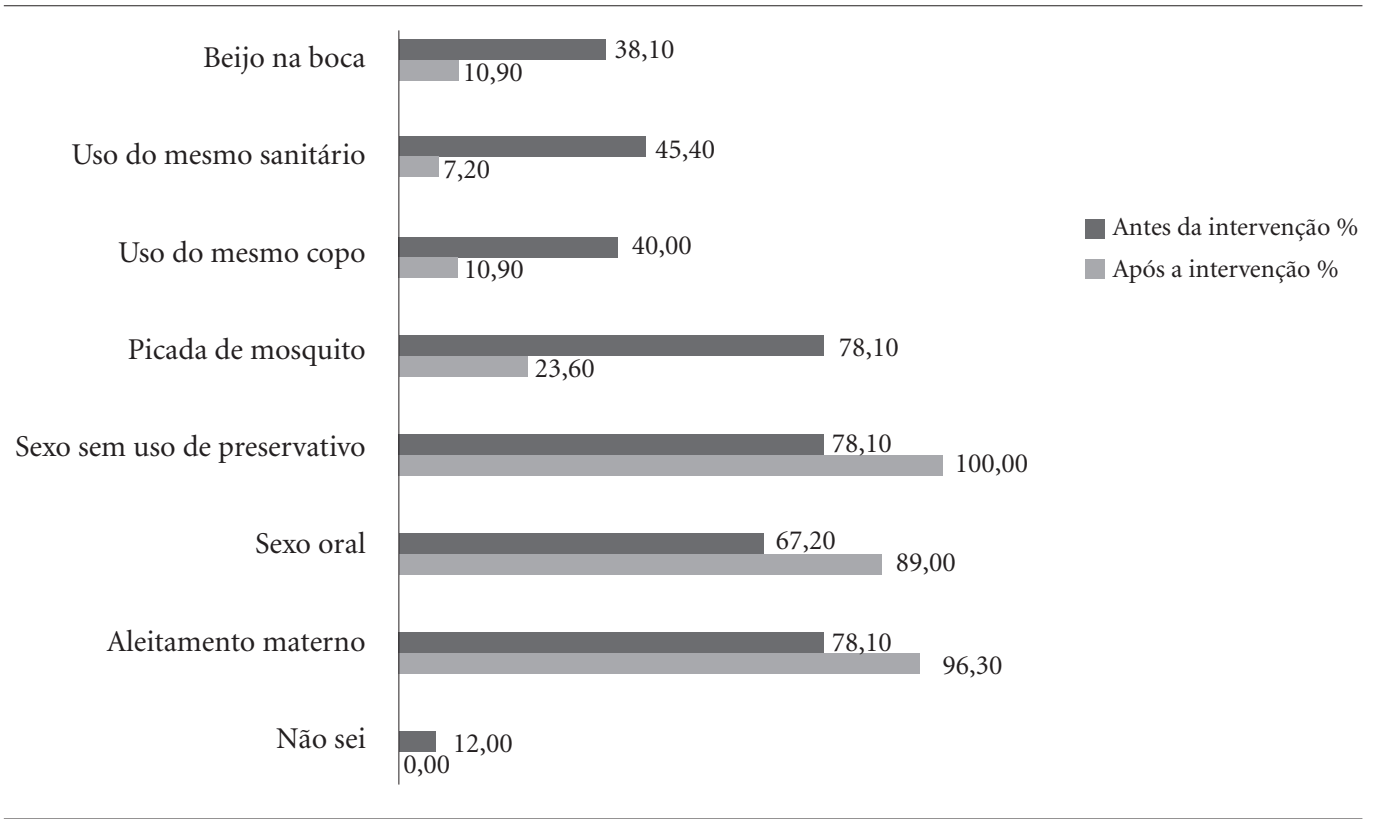

Gráfico 1. Distribuição da percepção dos idosos sobre as formas de transmissão do HIV, antes e depois da realização de intervenções educativas. Sobral, CE, 2014. 
Tabela 3. Média de conhecimento dos idosos antes e após a realização das oficinas. Sobral, CE, 2014.

\begin{tabular}{lccccc}
\hline \multicolumn{1}{c}{ Variável } & $\mathbf{n}$ & $\bar{x}$ & DP & p & t \\
\hline Conhecimento antes oficinas & 55 & 11,7 & 3,9 & & \\
Conhecimento após oficinas & 55 & 17,3 & 2,2 & $<0,001$ & $-8,8$ \\
\hline
\end{tabular}

\section{Discussão}

A análise da distribuição sociodemográfica dos indivíduos idosos atendidos no CSF Terrenos Novos em Sobral - CE demonstrou que a maioria era aposentados, com renda mensal de 1 salário mínimo. Segundo Silva et al. ${ }^{16}$, a presença de renda tem reflexo positivo sobre o envelhecimento ativo, pois o idoso contribui com orçamento familiar e possui independência financeira, diante de suas necessidades. No entanto também se deve considerar que a população é predominantemente de baixa renda, que muitas vezes se encontra associado ao baixo nível de escolaridade, fato que torna o indivíduo mais vulnerável ${ }^{17}$. Outro aspecto importante observado refere-se à predominância de mulheres, o qual se apresenta em consonância com o panorama de feminização do envelhecimento, visto que elas apresentam uma sobrevida de aproximadamente cinco anos superior ao dos homens ${ }^{15,18,19}$. Tal fato também se deve à característica de as mulheres procurarem com maior frequência o serviço de saúde, em relação ao homem. Levorato et al. ${ }^{20}$ e Brasil ${ }^{21}$ ressaltaram que a quantidade de mulheres que procuram por assistência à saúde é aproximadamente 2 vezes maior do que a de homens.

A configuração demográfica da pesquisa representa o atual perfil de idosos usuários da rede pública de saúde que, de acordo com Medeiros et al. ${ }^{15} \mathrm{e}$ Nascimento et al. ${ }^{17}$, segue a mesma tendência da conjuntura, na qual as epidemias de Aids e outras IST podem se expandir.

Em relação a Aids, uma doença infectocontagiosa causada pelo vírus HIV, a maioria dos entrevistados sabia do que a doença se tratava.
Nossos achados corroboram os dados obervados na pesquisa de Asaduzzaman et al..$^{22}$ em que cerca de dois terços dos entrevistados já tinha conhecimento sobre HIV/Aids.

Entretanto, quanto à forma de transmissão foi possível observar a existência de mitos e verdades decorrentes das dimensões culturais ou simbólicas da Aids, fato que gera preconceito em relação as pessoas que vivem com a HIV/Aids ${ }^{23}$. Antes das oficinas observou-se que uma parcela significativa dos idosos apontou a picada de mosquito, uso do mesmo sanitário e do mesmo copo como meio de transmissão do HIV. Resultados similares foram encontrados Lazzarotto et al..$^{24} \mathrm{e}$ Nascimento et al. ${ }^{17}$, em que $41,4 \%$, e $47,4 \%$ dos entrevistados, respectivamente, consideraram que mosquitos fossem vetores de transmissão do HIV.

Sabe-se que a Aids, assim como a sífilis é transmitida através das vias sexual, sanguínea, parenteral, ocupacional e vertical ${ }^{10}$. Os insetos não são vetores de transmissão do HIV, devido à ausência de receptores $\mathrm{CD} 4^{+}$nas células e à baixa taxa de infectividade e sobrevida do HIV fora do organismo humano $0^{25}$. Durante a oficina foi elucidado que mosquitos não eram vetores do HIV, no entanto estavam relacionados à transmissão de outras doenças, não menos graves, como dengue e leishmaniose visceral que também mereciam atenção especial quanto à prevenção.

No quesito tratamento, ainda que a maioria dos idosos tenha respondido corretamente que a Aids não tem cura, outra parcela significativa relatou não saber informar. Nas oficinas foi então esclarecido que apesar de não ter cura, existe tratamento, por meio do uso de medicamentos 
que atuam tornando mais lenta a progressão da doença e melhorando a qualidade de vida de pes-

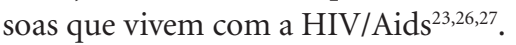

Quanto à sífilis, apesar de ser uma IST com maior prevalência na população jovem e adulta, têm sido observadas altas taxas de incidência no bairro dos Terrenos Novos em Sobral, e a maioria dos idosos afirmou não conhecê-la e não saber a sua forma de transmissão. A mesma trata-se de uma doença infecto-contagiosa de evolução crônica causada pela bactéria Treponema pallidum e caracterizada por episódios de atividade clínica ativa, intercaladas por períodos de infecção latente ${ }^{10}$.

O pouco conhecimento em relação à sífilis adquirida poderia ser explicado pela menor abordagem da mesma na estratégia da saúde da família. Uma vez que a grande preocupação dos serviços de saúde se concentra em torno do diagnóstico e tratamento da sífilis gestacional e congênita, sendo que esta poderia ser prevenida através da aquisição do conhecimento sobre a doença. Tal fato pode ser reafirmado, uma vez que após a realização das oficinas evidenciou-se uma mudança significativa no padrão de respostas, sendo que 98\% dos entrevistados responderam corretamente que o ato sexual sem preservativo era o principal meio de transmissão.

Outro fator importante observado foi em relação à possibilidade de cura da sífilis, antes das oficinas, a maioria desconhecia a forma de tratamento da sífilis. Estes resultados corroboram com Brêtas et al. ${ }^{28}$ no qual $12 \%$ dos entrevistados afirmaram que nenhuma das IST tem cura, e $48 \%$ não souberam responder. A sífilis assim como outras IST é um problema de saúde pública por sua magnitude e pela dificuldade das pessoas identificarem seus sintomas. O tratamento imediato das IST é, portanto, de importância fundamental, pois as feridas, inflamações, corrimentos nos órgãos genitais são portas de entrada para outras IST, como Aids.

O tratamento da sífilis se faz com uso de penicilina benzatina que age interferindo na síntese do peptidoglicano, componente da parede celular do T. pallidum. O esquema de dosagem e intervalo de aplicação depende do estágio da doença e tempo de evolução sendo de fundamental importância segui-lo rigorosamente para atingir a cura da doença ${ }^{29}$.

No quesito prevenção, os idosos, tanto antes quanto após as oficinas educativas, indicaram a relação sexual desprotegida como principal meio de transmissão da Aids e sífilis. Entretanto, a maioria dos entrevistados afirmou que não usava preservativo. Os índices foram maiores que aqueles encontrados nos estudos de Medeiros et al. ${ }^{15}$, Lazzarotto et al. ${ }^{24}$ e Prado et al. ${ }^{30}$, que correspondiam respectivamente a 46\%, 75\% e 76\%. Um dos motivos que podem justificar tal negligência é o fato de a maioria dos entrevistados serem mulheres que se encontram em período de pós-menopausa e, portanto, sem risco de engravidar, revelando a associação errônea do uso de preservativos com finalidade apenas de contracepção ${ }^{31,32}$.

Por outro lado, a resposta de não uso de preservativos pode refletir o fato de que apenas $29,69 \%$ dos entrevistados referiram ter vida sexual ativa e possuírem parceiro fixo e estável, fato que proporciona uma falsa "sensação de proteção" ${ }^{1,32}$. Zawacki et al. ${ }^{33}$ afirmou que longos anos de convivência traz consigo a familiaridade, sendo este um fator responsável pela negligência ao uso de camisinha, pois pode influenciar as percepções de risco de contaminação pelo viés de projeção levando ao pressuposto de que o parceiro sexual tem baixo risco de estar contaminado por alguma IST.

Além disso, o uso ou não de preservativo depende da negociação entre parceiros e muitas vezes da anuência dos homens. De acordo com Driemeier et al. ${ }^{34}$ homens com 50 anos ou mais apresentam comportamento sexual de alto risco, pois o uso de preservativo é seis vezes menor do que entre jovens do gênero masculino. O não uso de preservativo pelos homens deve-se ao fato de que eles acreditavam diminuir o prazer e prejudicar a ereção. Foi ainda referido que os homens não consideravam legítimo que as esposas solicitassem o uso da camisinha para prevenção da Aids, pois deveriam confiar nos maridos.

Outros fatores relacionados à resistência dessa faixa etária ao uso de preservativos, como a formação cultural correspondente à época, bem como a falta de conhecimento sobre IST e suas vias de transmissão que tornam esse grupo ainda mais vulnerável ${ }^{34}$. Em Albuquerque et al. ${ }^{35}$, observou-se menor conhecimento dos idosos sobre Aids, quando comparados aos jovens, perpassando o conceito da enfermidade, transmissibilidade, condutas preventivas, diagnóstico e tratamento, pois ofereceram maior número de respostas insatisfatórias.

Desta forma, no contexto gerontológico o conhecimento em saúde é um determinante para o risco comportamental e a percepção de vulnerabilidade, visto que qualquer faixa etária está vulnerável às infecções sexualmente transmissíveis, dependendo da situação de risco a que cada um está exposto. Nesse sentido, esta pesquisa 
mostrou vários resultados relevantes apesar das limitações devidas principalmente à amostragem e ao período curto entre a intervenção e a aferição do conhecimento, pode-se verificar a importância em reconhecer os valores e a cultura dos indivíduos envolvidos e promover campanhas dinâmicas e com direcionamentos diferentes ao público alvo. Objetiva-se, com isso, atingir resultados mais efetivos em termos de prevenção, de uma atividade sexual segura, promovendo saúde à população de forma mais equânime.

\section{Conclusão}

Apesar das limitações do estudo referente à amostragem e ao período curto entre a aferição do conhecimento, podem-se observar lacunas no conhecimento desse grupo com 60 ou mais anos de idade sobre conceito, transmissão, prevenção, vulnerabilidade e tratamento da Aids e sífilis. No entanto, a realização de oficinas educativas sobre HIV/Aids e sífilis demonstrou ser uma intervenção eficaz no que se refere à assimilação de conhecimento pelos idosos. $\mathrm{O}$ acolhimento e o diálogo foram elementos essenciais para a estruturação de um ambiente profícuo de intervenção no contexto da terceira idade. Além disso, a interdisciplinaridade, proporcionada pelo PET-Saúde, durante a abordagem contribuiu de forma importante para enriquecimento do conteúdo das oficinas e para formação profissional dos integrantes. Portanto, os resultados deste trabalho sugerem a necessidade do desenvolvimento regular de programas educativos, de forma integrada por profissionais e gestores em saúde, bem como a implementação de estratégias entre diferentes faixas etárias, visando à adoção de praticas preventivas e que sejam adequadas ao contexto de cada individuo.

\section{Colaboradores}

LM Bastos, JMS Tolentino e MAO Frota participaram na coleta e análise dos dados, no desenvolvimento das oficinas educativas e escrita do artigo. WC Tomaz participou na coleta, análise dos dados e no desenvolvimento das oficinas educativas. MLS Fialho colaborou na articulação dos idosos para participação da pesquisa e no desenvolvimento das atividades educativas. ACB Batista participou da elaboração do projeto de pesquisa e no desenvolvimento das atividades educativas. AKM Teixeira fez a análise estatística dos dados e revisou a escrita do artigo. FCB Barbosa participou da elaboração do projeto, enviou para Comitê de Ética, revisou escrita do artigo e supervisionou as atividades desenvolvidas.

\section{Referências}

1. Küchemann BA. Envelhecimento Populacional, cuidado e cidadania: velhos dilemas e novos desafios. Revista Sociedade e Estado 2012; 27(1):165-180.

2. Instituto Brasileiro de Geografia e Estatística (IBGE). Síntese de indicadores sociais: Uma análise das condições de vida da população brasileira. Rio de Janeiro. № 32. [acessado 2014 Jun 25]. Disponível em: http://biblioteca.ibge.gov.br/visualizacao/livros/liv66777.pdf

3. Brunnet AE, Andrades B, Souza CS, Weber JLA, Martinato L, Loreto T, Pizzinato A. Práticas sociais e significados do envelhecimento para mulheres idosas. Pensando Famílias 2013; 17(1):99-109.

4. Guerra ACLC, Caldas CP. Dificuldades e recompensas no processo de envelhecimento: a percepção do sujeito idoso. Cien Saude Colet 2010; 15(6):2931-2940.

5. Organização Mundial da Saúde (OMS). 2015 [acessado 2015 Jun 07]. Disponível em: http://www.who.int/em

6. Araújo VLB, Brito DMS, Gimeniz MT, Queiroz TA, Tavares CM. Características da Aids na terceira idade em um hospital de referência do Estado do Ceará, Brasil. Rev Bras Epidemiol 2007; 10(4):544-554.

7. Oliveira MLC, Paz LC, Melo GF. Dez anos de epidemia do HIVAIDS em maiores de 60 anos no Distrito Federal - Brasil. Rev Bras Epidemiol 2013; 16(1):30-39.

8. Brasil. Ministério da Saúde (MS). Boletim Epidemiológico - HIV e AIDS. Brasília: MS. [acessado 2014 Maio 15]. Disponível em: http://www.aids.gov.br/publicacao/2015/boletimepidemiologico-aids-e-dst-2015 
9. Ceará. Secretaria Estadual de Saúde. Coordenadoria de Promoção e Proteção à Saúde - Núcleo de Epidemiologia - SESA/CE. Informe Epidemiológico, novembro, 2013. [acessado 2014 Abr 27]. Disponível em: http:// www.saude.ce.gov.br/index.php/boletins\#

10. Garcia FLB. Prevalência de sifilis em adolescentes e jovens do sexo feminino noestado de Goiás [dissertação]. Goiânia: Universidade Federal de Goiás; 2009.

11. Brasil. Ministério da Saúde (MS). Guia de Bolso - Doenças parasitária e infecciosas. 2005. [acessado 2012 Out 20]. Disponível em: http://bvsms.saude.gov.br/bvs/publicacoes/guia_bolso_5ed2.pdf

12. Pereira GS, Borges CI. Conhecimento sobre HIV/AIDS de participantes de um grupo de idosos em Anápolis, Goiás. Esc Anna Nery 2010; 14(4):720-725.

13. Godoy VS, Ferreira MD, Silva EC, Gir E, Canini SRMS. O perfil epidemiológico da AIDS em idosos utilizando sistema de informações em saúde do Datasus: Realidade e desafios. J Bras Doenças Sex Transm 2008; 20(1):7-11.

14. Camargo BV, Torres TL, Biasus F. Práticas sexuais, conhecimento sobre HIV/AIDS e atitudes a respeito da relação amorosa e prevenção entre adultos com mais de 50 anos do sul do Brasil. Liberat 2009; 15(2):171-180.

15. Medeiros KCS, Leal MCC, Marques APO, Marino JG. Avaliação do nível de informação em relação à Aids/ HIV por idosos assistidos no Programa de Saúde da Família. Geriatria \& Gerontologia 2008; 2(2):53-58.

16. Silva AA, Souza MR, Flores MFS, Lima NB. AIDS na terceira idade: uma revisão da literatura [dissertação]. Governador Valadares: Universidade Vale do Rio Doce; 2009.

17. Nascimento RG, Monteiro EL, Ferreira ES, Santos ZNL. Nível de conhecimento de idosos comunitários em relação ao HIV/Aids: estudo exploratório na rede básica de saúde de Belém, Pará, Brasil. RBCEH 2013; 10(1):113-122.

18. Souza MHT, Backes DS, Pereira ADA, Ferreira CLL, Medeiros HMF, Marchiori MRCT. Nível de conhecimento de um grupo de idosos em relação à Síndrome da Imunodeficiência Adquirida. Rev Avanços em Enfermagem 2009; 27(1):22-29.

19. Pilger C, Menon MH, Mathias TAF. Características sociodemográficas e de saúde de idosos: contribuições para os serviços de saúde. Rev. Latino-Am. Enfermagem 2011; 19(5):1-9.

20. Levorato CD, Mello LM, Silva AS, Nunes AA. Fatores associados à procura por serviços de saúde numa perspectiva relacional de gênero. Cien Saude Colet 2014; 19(4):1263-1274.

21. Brasil. Ministério da Saúde (MS). Secretaria de Vigilância em Saúde. Pesquisa de conhecimentos, atitudes e práticas relacionada à DST e Aids da população brasileira de 15 a 64 anos de idade. 2008. [acessado 2016 Jul 10]. Disponível em: http://www.aids. gov.br/sites/default/ files/PCAP-BR_CNAIDS.ppt

22. Asaduzzaman M, Higuchi M, Sarker MAB, Hamajima $\mathrm{N}$. Awareness and knowledge of HIV/AIDS among married women in rural Bangladesh and exposure to media: a secondary data analysis of the 2011 Bangladesh Demographic and Health Survey. Nagoya J Med Sci 2016; 78(1):109-118.

23. Lima MM, Carlos J, Areal RB, Souza RJS, Lima SS, Campos LAO, Eisenlohr PV, Filardi MP. Conhecimento da população de Viçosa, MG, sobre as formas de transmissão da AIDS. Cien Saude Colet 2008; 13(6):18791888 .
24. Lazzarotto AR, Kramer AS, Hädrich M, Tonin M, Caputo P, Sprinz E. O conhecimento de HIV/aids na terceira idade: estudo epidemiológico no Vale do Sinos, Rio Grande do Sul, Brasil. Cien Saude Colet 2008; 13(6):1833-1840.

25. Iqbal MM. Can we get AIDS from mosquito bites? J La State Med Soc 1999; 151(8):429-433.

26. Galvão MTG, Soares LL, Pedrosa SC, Fiuza MLT, Lemos LA. Qualidade de vida e adesão à medicação antirretroviral em pessoas com HIV. Acta Paul Enferm 2015; 28(1):48-53.

27. Pimenta CJL, Costa IP, Farias MCAD, Costa KNFM, Pereira TLB, Leite ES, Moraes JCO, Silva CR, Almeida MR, Vasconcelos DA, Sousa ASQ, Costa TF. HIV/AIDS and Seniors: Knowledge of the Elderly about the Disease. International Archives of Medicine 2016; 20(9):1-7.

28. Brêtas JRS, Ohara CVS, Jardim DP, Muroya RL. Conhecimentos de adolescentes sobre Doenças Sexualmente Transmissíveis: subsídios para prevenção. Acta Paul Enferm 2009; 22(6):786-792.

29. Brasil. Ministério da Saúde (MS). Diretrizes para controle de Sifilis congênita -Manual de Bolso. 2006. [acessado 2015 Set 27]. Disponível em: http://bvsms.saude. gov.br/bvs/publicacoes/manual_sifilis_bolso.pdf

30. Prado DJ, Neves JEF, Silva GS, Silva ICR. O conhecimento de HIV/AIDS em idosos de uma comunidade carente do Distrito Federal. Acta de Ciências e Saúde 2012; 1(2):87-101.

31. Olivi M, Santana RG, Mathias TAF. Comportamento, conhecimento e percepção de risco sobre doenças sexualmente transmissíveis em um grupo de pessoas com 50 anos ou mais de idade. Rev Latino Enfermagem 2008; $16(4): 1-8$.

32. Laroque MF, Affeldt AB, Cardoso DH, Souza GL, Santana MG, Lange C. Sexualidade do idoso: comportamento para a prevenção de DST/ AIDS. Rev Gaúcha Enferm 2011; 32(4):774-80.

33. Zawacki T, Norris J, Hessler DM, Morrison DM, Stoner SA, George WH, Davis KC, Abdallah DA. Effects of relationship motivation, partner familiarity, and alcohol on women's risky sexual decision making. Pers Soc Psychol Bull 2009; 35(6):723-736.

34. Driemeier M, Andrade SMO, Pontes ERJC, Paniago AMM, Cunha RV. Vulnerability to AIDS among the elderly in an urban center in central Brazil. Clinics 2012; 67(1):19-25.

35. Albuquerque HMM, Leal MCC, Oliveira APM, Marino JG. O conhecimento sobre Aids de homens idosos e adultos jovens: um estudo sobre a percepção desta doença. Cien Saude Colet 2012; 17(1):43-53.

Artigo apresentado em 07/10/2015

Aprovado em 23/08/2016

Versão final apresentada em 25/08/2016 